\title{
Muon-spin rotation measurements of the magnetic penetration depth in the iron-based superconductor $\mathrm{Ba}_{1-x} \mathrm{Rb}_{x} \mathrm{Fe}_{2} \mathrm{As}_{2}$
}

\author{
Z. Guguchia, ${ }^{1,}$ Z. Shermadini, ${ }^{2}$ A. Amato, ${ }^{2}$ A. Maisuradze,,${ }^{1,2}$ A. Shengelaya, ${ }^{3}$ Z. Bukowski, ${ }^{4,5}$ \\ H. Luetkens, ${ }^{2}$ R. Khasanov, ${ }^{2}$ J. Karpinski, ${ }^{4}$ and H. Keller ${ }^{1}$ \\ ${ }^{1}$ Physik-Institut der Universität Zürich, Winterthurerstrasse 190, CH-8057 Zürich, Switzerland \\ ${ }^{2}$ Laboratory for Muon Spin Spectroscopy, Paul Scherrer Institute, CH-5232 Villigen PSI, Switzerland \\ ${ }^{3}$ Department of Physics, Tbilisi State University, Chavchavadze 3, GE-0128 Tbilisi, Georgia \\ ${ }^{4}$ Laboratory for Solid State Physics, ETH Zürich, CH-8093 Zürich, Switzerland \\ ${ }^{5}$ Institute of Low Temperature and Structure Research, Polish Academy of Sciences, 50-422 Wroclaw, Poland
}

(Received 13 June 2011; published 14 September 2011)

\begin{abstract}
Measurements of the magnetic penetration depth $\lambda$ in the Fe-based superconductor $\mathrm{Ba}_{1-x} \mathrm{Rb}_{x} \mathrm{Fe}_{2} \mathrm{As}_{2}(x=0.3$, $0.35,0.4)$ were carried out using the muon-spin rotation $(\mu \mathrm{SR})$ technique. The temperature dependence of $\lambda$ is well described by a two-gap $s+s$-wave scenario with a small gap $\Delta_{1} \approx 1-3 \mathrm{meV}$ and a large gap $\Delta_{2} \approx$ 7-9 meV. By combining the present data with those previously obtained for $\mathrm{RbFe}_{2} \mathrm{As}_{2}$ a decrease of the BCS ratio $2 \Delta_{2} / k_{\mathrm{B}} T_{\mathrm{c}}$ with increasing Rb content $x$ is observed. On the other hand, the BCS ratio $2 \Delta_{1} / k_{\mathrm{B}} T_{\mathrm{c}}$ is almost independent of $x$. In addition, the contribution of $\Delta_{1}$ to the superfluid density is found to increase with $x$. These results are discussed in light of the suppression of interband processes upon hole doping.
\end{abstract}

DOI: 10.1103/PhysRevB.84.094513

PACS number(s): 74.20.Mn, 74.25.Ha, 74.70.Xa, 76.75.+i

\section{INTRODUCTION}

The discovery ${ }^{1}$ of superconductivity in iron oxypnictide $\mathrm{LaFeAsO}_{1-x} \mathrm{~F}_{x}$ has generated great interest in the phenomenon of high temperature superconductivity. The basic units responsible for superconductivity are the fluorite type $\left[\mathrm{Fe}_{2} \mathrm{Pn}_{2}\right]$ layers where $P n$ is a pnictogen element (P, As, $\mathrm{Sb}$, and $\mathrm{Bi}$ ). These layers are separated by spacer layers which play the role of a charge reservoir. In the fluorite-type layers the $\mathrm{Fe}$ atoms are surrounded by four pnictogen atoms forming a tetrahedron. The first class of iron-based superconductors studied has the $\mathrm{ZrCuSiAs}$ structure (1111 compounds), where the spacer layer $\left[L n_{2} \mathrm{O}_{2}\right]$ has the "antifluoride" or $\mathrm{Pb}_{2} \mathrm{O}_{2}$ structure. With $L n=\mathrm{Sm}$ a critical temperature higher than $55 \mathrm{~K}$ was observed. ${ }^{2}$

Superconductivity with $T_{\mathrm{c}}=38 \mathrm{~K}$ was also found in the ternary systems $A \mathrm{Fe}_{2} \mathrm{As}_{2}$ (Refs. 3 and 4) (122 compounds) adopting the tetragonal $\mathrm{ThCr}_{2} \mathrm{Si}_{2}$ structure. In this structure the spacer layer is provided by an alkali earth element $A=$ $\mathrm{Ca}, \mathrm{Sr}$, or Ba. Doping is realized by the substitution of $A$ by an alkali metal such as K, Cs, or Rb. Several disconnected Fermisurface sheets contribute to superconductivity as revealed by angle-resolved photoemission spectroscopy (ARPES).$^{5-7}$ Moreover, indications of multigap superconductivity in the system $\mathrm{Ba}_{1-x} \mathrm{~K}_{x} \mathrm{Fe}_{2} \mathrm{As}_{2}$ were obtained from the temperature dependence of the magnetic penetration depth $\lambda$ by means of muon-spin rotation $(\mu \mathrm{SR})^{8}$ and ARPES. ${ }^{5}$ The magnetic penetration depth is one of the fundamental parameters of a superconductor since it is closely related to the density of the superconducting carriers $n_{s}$ and their effective mass $m^{*}$ via the relation $1 / \lambda^{2} \propto n_{s} / m^{*}$. The temperature dependence of $\lambda$ reflects the topology of the superconducting gap occurring in the density of states of the superconducting ground state. The $\mu$ SR technique provides a powerful tool to measure $\lambda$ in type II superconductors. ${ }^{9}$

As demonstrated in previous works, ${ }^{4,10}$ the value of $T_{\mathrm{c}}$ for hole-doped $\mathrm{Ba}_{1-x} \mathrm{Rb}_{x} \mathrm{Fe}_{2} \mathrm{As}_{2}$ decreases monotonically upon increasing the $\mathrm{Rb}$ content $x$ in the overdoped region. However, in contrast to the overdoped cuprates, $T_{\mathrm{c}}$ remains finite even at the highest doping level $x=1$ with $T_{\mathrm{c}}=2.52 \mathrm{~K}$ (Ref. 4). A detailed study of the doping dependence of $T_{\mathrm{c}}$ may help to clarify the origin of high- $T_{\mathrm{c}}$ superconductivity in these iron-based systems. It is thus of importance to investigate the superconducting properties of optimally doped $\mathrm{Ba}_{1-x} \mathrm{Rb}_{x} \mathrm{Fe}_{2} \mathrm{As}_{2}$ and compare the results with those obtained for $\mathrm{RbFe}_{2} \mathrm{As}_{2}$ (Ref. 10).

In this paper, we report on $\mu \mathrm{SR}$ studies of the temperature and field dependence of the magnetic penetration depth of optimally doped $\mathrm{Ba}_{1-x} \mathrm{Rb}_{x} \mathrm{Fe}_{2} \mathrm{As}_{2}(x=0.3,0.35,0.4)$. We compare the present data with the previous results of overdoped $\mathrm{RbFe}_{2} \mathrm{As}_{2}$ (Ref. 10) and discuss the combined results in light of the suppression of interband processes upon hole doping.

\section{EXPERIMENTAL DETAILS}

Polycrystalline samples of $\mathrm{Ba}_{1-x} \mathrm{Rb}_{x} \mathrm{Fe}_{2} \mathrm{As}_{2}$ were prepared in evacuated quartz ampoules by a solid state reaction method. $\mathrm{Fe}_{2} \mathrm{As}$, BaAs, and $\mathrm{RbAs}$ were obtained by reacting high purity As $(99.999 \%), \mathrm{Fe}(99.9 \%), \mathrm{Ba}(99.9 \%)$, and $\mathrm{Rb}(99.95 \%)$ at $800{ }^{\circ} \mathrm{C}, 650{ }^{\circ} \mathrm{C}$, and $500{ }^{\circ} \mathrm{C}$, respectively. Using stoichiometric amounts of BaAs or RbAs and $\mathrm{Fe}_{2} \mathrm{As}$ the terminal compounds $\mathrm{BaFe}_{2} \mathrm{As}_{2}$ and $\mathrm{RbFe}_{2} \mathrm{As}_{2}$ were synthesized at $950{ }^{\circ} \mathrm{C}$ and $650{ }^{\circ} \mathrm{C}$, respectively. Finally, the samples of $\mathrm{Ba}_{1-x} \mathrm{Rb}_{x} \mathrm{Fe}_{2} \mathrm{As}_{2}$ with $x=0.3$, 0.35, 0.4 were prepared from appropriate amounts of single-phase $\mathrm{BaFe}_{2} \mathrm{As}_{2}$ and $\mathrm{RbFe}_{2} \mathrm{As}_{2}$. The components were mixed, pressed into pellets, placed into alumina crucibles, and annealed for 100 hours at $650{ }^{\circ} \mathrm{C}$ with one intermittent grinding. Powder $\mathrm{X}$-ray diffraction analysis revealed that the synthesized samples are single phase materials. Zero-field (ZF) and transverse-field (TF) $\mu \mathrm{SR}$ experiments were performed at the $\pi \mathrm{M} 3$ beamline of the Paul Scherrer Institute (Villigen, Switzerland), using the general purpose instrument (GPS). The sample was mounted inside of a gas-flow ${ }^{4} \mathrm{He}$ cryostat on a sample holder with a standard veto setup providing essentially a low-background 
$\mu \mathrm{SR}$ signal. All TF experiments were carried out after a field-cooling procedure.

\section{RESULTS AND DISCUSSION}

Figures 1(a) and 1(b) exhibit the transverse-field (TF) muon-time spectra for $\mathrm{Ba}_{1-x} \mathrm{Rb}_{x} \mathrm{Fe}_{2} \mathrm{As}_{2}(x=0.3,0.4)$ measured in an applied magnetic field of $\mu_{0} H=0.04 \mathrm{~T}$ above $(45 \mathrm{~K})$ and below $(1.7 \mathrm{~K})$ the superconducting $(\mathrm{SC})$ transition temperature $T_{\mathrm{c}}$. Above $T_{\mathrm{c}}$ the oscillations show a small relaxation due to the random local fields from the nuclear magnetic moments. Below $T_{\mathrm{c}}$ the relaxation rate strongly increases due to the presence of a nonuniform local field distribution as a result of the formation of a flux-line lattice (FLL) in the SC state. It is well known that undoped $\mathrm{BaFe}_{2} \mathrm{As}_{2}$ is not superconducting at ambient pressure and undergoes a spin-density wave (SDW) transition of the Fe moments far above $T_{\mathrm{c}}$ (Ref. 11). The SC state can be achieved either under pressure ${ }^{12,13}$ or by appropriate charge carrier doping ${ }^{14}$ of the parent compounds, leading to a suppression of the
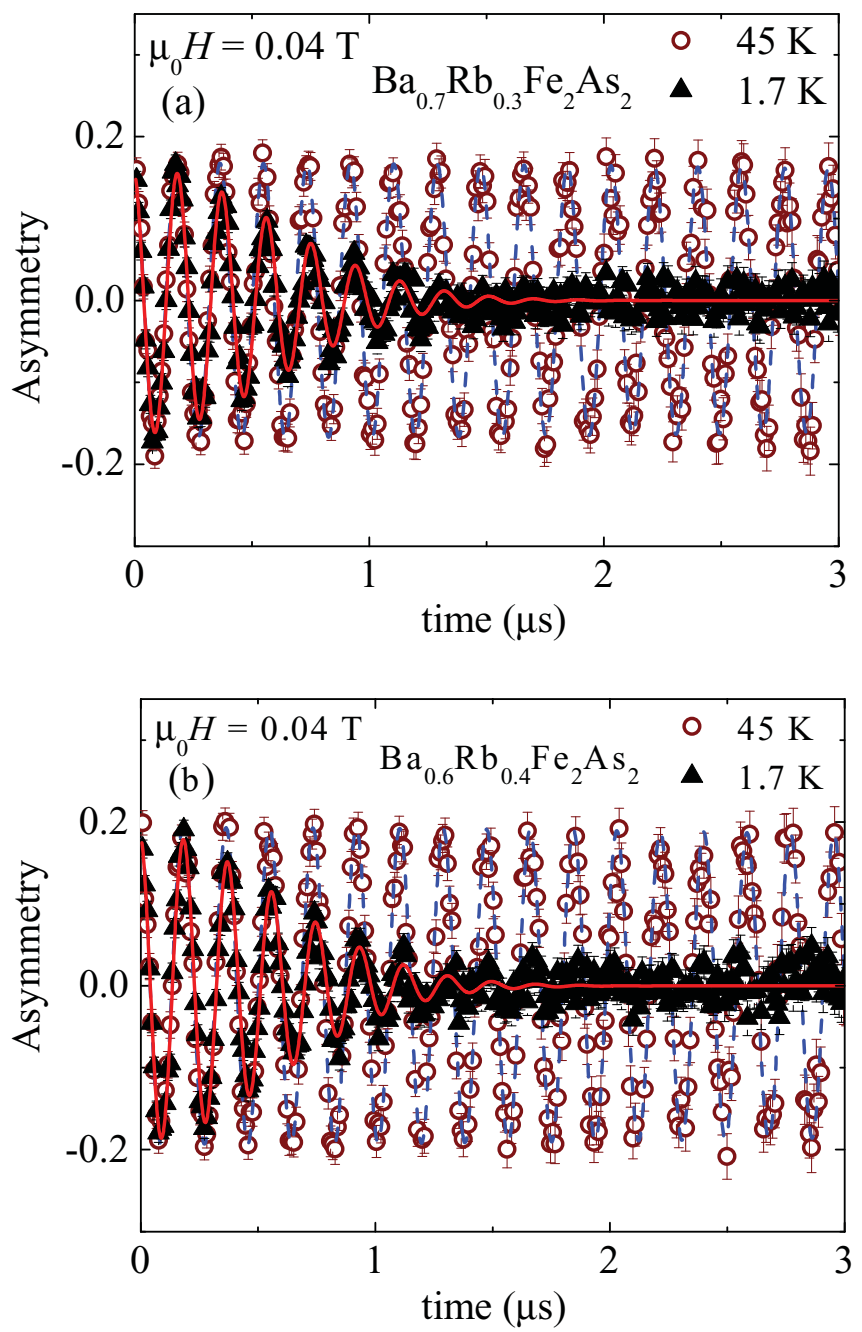

FIG. 1. (Color online) Transverse field (TF) $\mu$ SR time spectra obtained in $\mu_{0} H=0.04 \mathrm{~T}$ above and below $T_{\mathrm{c}}$ (after field cooling the sample from above $T_{\mathrm{c}}$ ): (a) $\mathrm{Ba}_{0.7} \mathrm{Rb}_{0.3} \mathrm{Fe}_{2} \mathrm{As}_{2}$ and (b) $\mathrm{Ba}_{0.6} \mathrm{Rb}_{0.4} \mathrm{Fe}_{2} \mathrm{As}_{2}$. The solid and the dashed lines represent fits to the data by means of Eq. (1).
SDW state. Magnetism, if present in the samples, may enhance the muon depolarization rate and falsify the interpretation of the TF- $\mu$ SR results. Therefore, we have carried out $\mathrm{ZF}-\mu \mathrm{SR}$ experiments above and below $T_{\mathrm{c}}$ to search for magnetism (static or fluctuating) in $\mathrm{Ba}_{1-x} \mathrm{Rb}_{x} \mathrm{Fe}_{2} \mathrm{As}_{2}$ ( $x=0.3,0.35,0.4$ ). As shown in Fig. 2(a) no sign of either static or fluctuating magnetism could be detected in $\mathrm{ZF}$ time spectra down to $1.7 \mathrm{~K}$. Moreover, the $\mathrm{ZF}$ relaxation rate is small and changes very little between 45 and $1.7 \mathrm{~K}$. The spectra are well described by a standard Kubo-Toyabe depolarization function, ${ }^{15}$ reflecting the field distribution at the muon site created by the nuclear moments.

It was reported ${ }^{16-18}$ that in some iron-based superconductors $\mathrm{BaFe}_{2-x} \mathrm{Co}_{x} \mathrm{As}_{2}$ and $\mathrm{SrFe}_{2-x} \mathrm{Co}_{x} \mathrm{As}_{2}$ field induced magnetism exists. In the present work TF- $\mu$ SR spectra measured in different applied fields (see Fig. 1 for $\mu_{0} H=$ $0.04 \mathrm{~T}$ ) exhibit a Gaussian-like depolarization above and below $T_{\mathrm{c}}$ that is typical of nuclear moments and the vortex lattice in polycrystalline samples, respectively. In the presence of dilute or fast fluctuating electronic moments one expects an
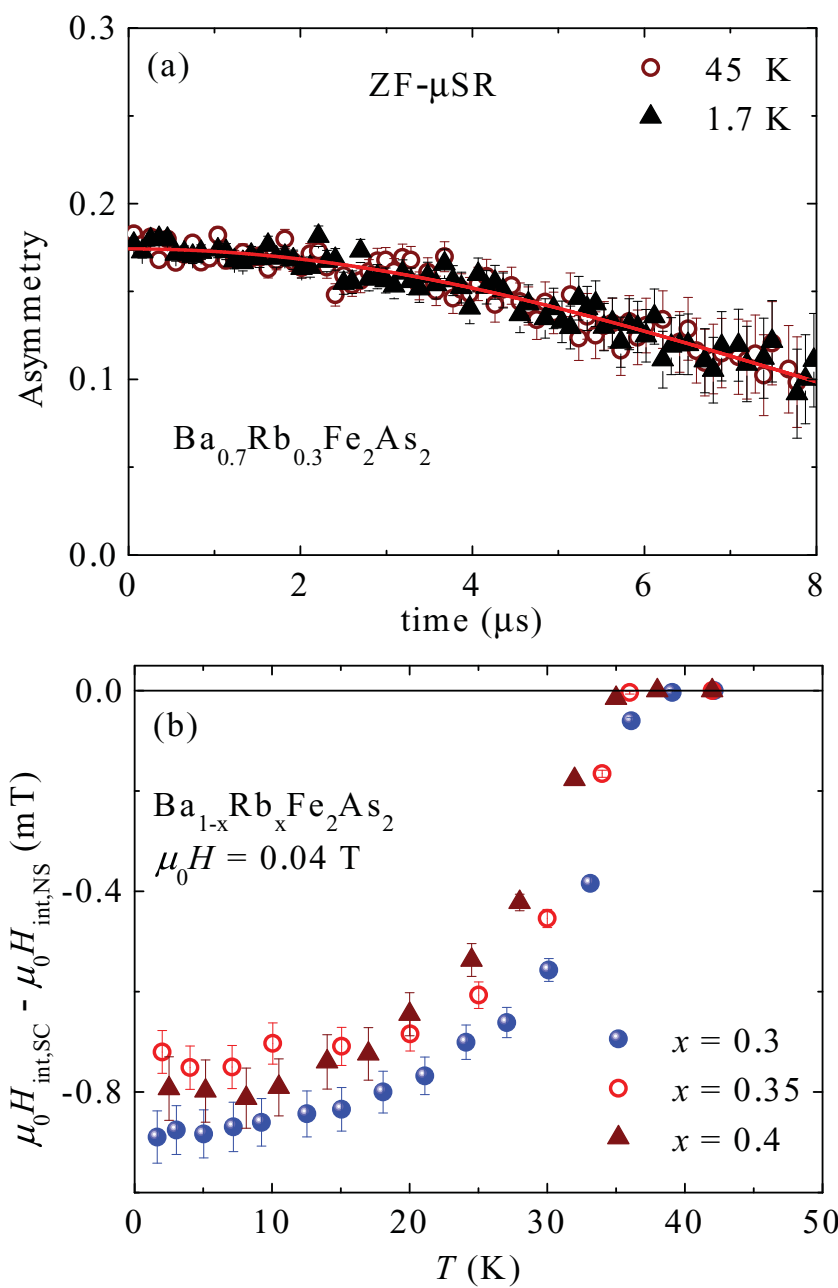

FIG. 2. (Color online) (a) ZF- $\mu$ SR time spectra for $\mathrm{Ba}_{0.7} \mathrm{Rb}_{0.3}$ $\mathrm{Fe}_{2} \mathrm{As}_{2}$ recorded above and below $T_{\mathrm{c}}$. The line represents the fit to the data of a standard Kubo-Toyabe depolarization function (Ref. 15). (b) Temperature dependence of the difference between the internal field $\mu_{0} H_{\text {int,SC }}$ measured in the SC state and the one measured in the normal state $\mu_{0} H_{\text {int,NS }}$ at $T=42 \mathrm{~K}$. 

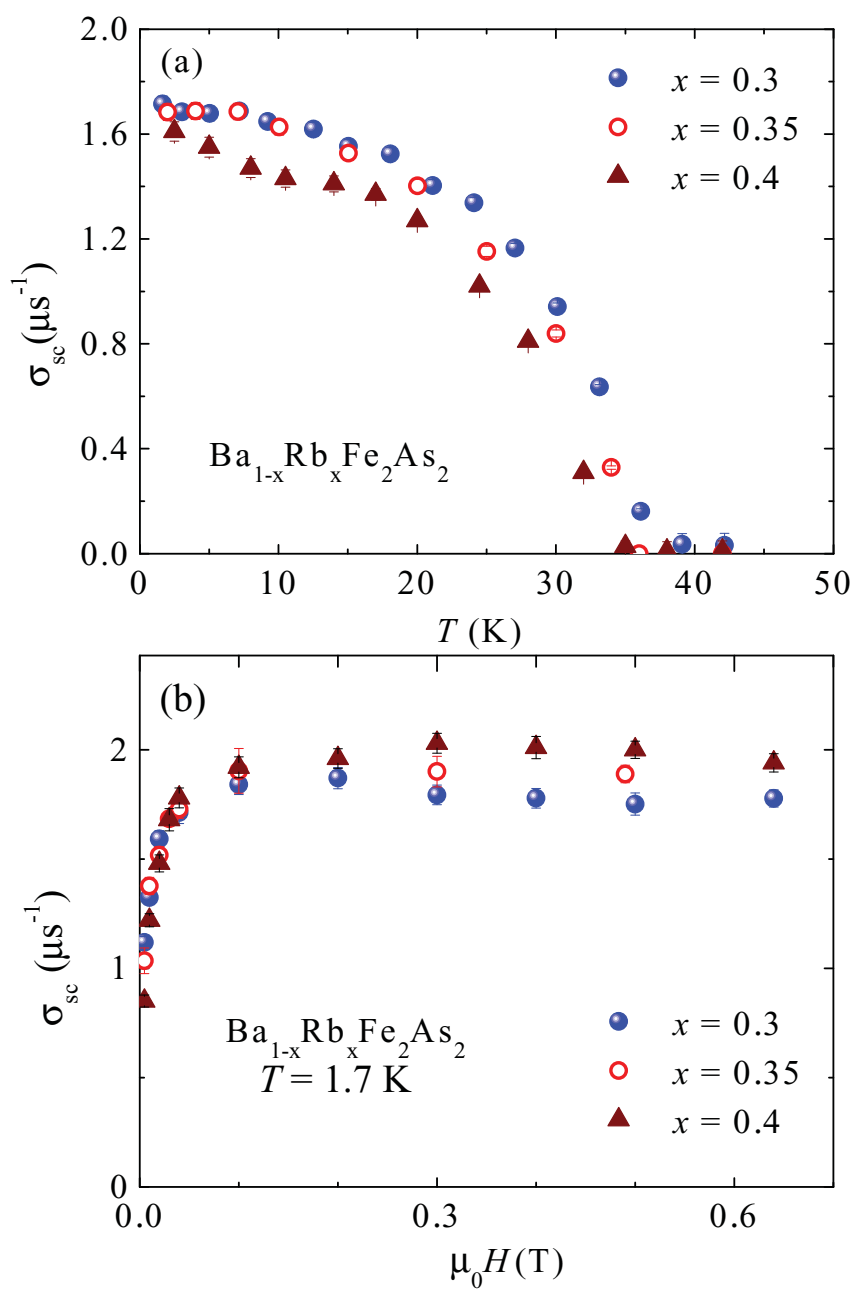

FIG. 3. (Color online) (a) Temperature dependence of the superconducting muon spin depolarization rate $\sigma_{\mathrm{sc}}$ measured in an applied magnetic field of $\mu_{0} H=0.04 \mathrm{~T}$ for $\mathrm{Ba}_{1-x} \mathrm{Rb}_{x} \mathrm{Fe}_{2} \mathrm{As}_{2}(x=0.3,0.35$, $0.4)$. (b) Field dependence of $\sigma_{\mathrm{sc}}$ at $1.7 \mathrm{~K}$.

exponential depolarization of the TF- $\mu$ SR spectrum, which is absent in the present case. Moreover, the SC muon depolarization rate $\sigma_{\mathrm{sc}}$ is constant at high fields as shown in Fig. 3(b). In addition we observed a diamagnetic shift of the internal magnetic field $\mu_{0} H_{\text {int }}$ sensed by the muons below $T_{\mathrm{c}}$. This is evident in Fig. 2(b), where we plot the difference between the internal field $\mu_{0} H_{\mathrm{int}, \mathrm{SC}}$ measured in the SC state and one $\mu_{0} H_{\text {int, NS }}$ measured in the normal state at $T=42 \mathrm{~K}$. Note, that in the systems $\mathrm{BaFe}_{2-x} \mathrm{Co}_{x} \mathrm{As}_{2}$ and $\mathrm{SrFe}_{2-x} \mathrm{Co}_{x} \mathrm{As}_{2}$, where the field induced magnetism was detected, paramagnetic shift was observed ${ }^{16-18}$ instead of the expected diamagnetic shift imposed by the SC state. All these observations indicate that there is no field induced magnetism in the system $\mathrm{Ba}_{1-x} \mathrm{Rb}_{x} \mathrm{Fe}_{2} \mathrm{As}_{2}$ down to $1.7 \mathrm{~K}$. The absence of magnetism in $\mathrm{Ba}_{1-x} \mathrm{Rb}_{x} \mathrm{Fe}_{2} \mathrm{As}_{2}$ implies that the increase of the TF relaxation rate below $T_{\mathrm{c}}$ is attributed entirely to the vortex lattice.

The TF $\mu$ SR data were analyzed by using the following functional form: ${ }^{19}$

$$
P(t)=A \exp \left[-\frac{\left(\sigma_{\mathrm{sc}}^{2}+\sigma_{\mathrm{nm}}^{2}\right) t^{2}}{2}\right] \cos \left(\gamma_{\mu} B_{\mathrm{int}} t+\varphi\right) .
$$

Here $A$ denotes the initial asymmetry, $\gamma /(2 \pi) \simeq$ $135.5 \mathrm{MHz} / \mathrm{T}$ is the muon gyromagnetic ratio, and $\varphi$ is the initial phase of the muon-spin ensemble. $B_{\text {int }}$ represents the internal magnetic field at the muon site, and the relaxation rates $\sigma_{\mathrm{sc}}$ and $\sigma_{\mathrm{nm}}$ characterize the damping due to the formation of the FLL in the superconducting state and of the nuclear magnetic dipolar contribution, respectively. In the analysis $\sigma_{\mathrm{nm}}$ was assumed to be constant over the entire temperature range and was fixed to the value obtained above $T_{\mathrm{c}}$ where only nuclear magnetic moments contribute to the muon depolarization rate $\sigma$. As indicated by the solid lines in Fig. 1, the $\mu$ SR data are well described by Eq. (1). The temperature dependence of $\sigma_{\text {sc }}$ for $\mathrm{Ba}_{1-x} \mathrm{Rb}_{x} \mathrm{Fe}_{2} \mathrm{As}_{2}(x=0.3,0.35$, and 0.4$)$ at $\mu_{0} H=$ $0.04 \mathrm{~T}$ is shown in Fig. 3(a). Below $T_{\mathrm{c}}$ the relaxation rate $\sigma_{\mathrm{sc}}$ starts to increase from zero due to the formation of the FLL.

For polycrystalline samples the temperature dependence of the London magnetic penetration depth $\lambda(T)$ is related to the superconducting part of the Gaussian muon spin depolarization rate $\sigma_{\mathrm{sc}}(T)$ by the equation ${ }^{20}$

$$
\frac{\sigma_{\mathrm{sc}}^{2}(T)}{\gamma_{\mu}^{2}}=0.00371 \frac{\Phi_{0}^{2}}{\lambda^{4}(T)}
$$

where $\Phi_{0}=2.068 \times 10^{-15} \mathrm{~Wb}$ is the magnetic-flux quantum. Equation (2) is only valid when the separation between the vortices is smaller than $\lambda$. In this case, according to the London model $\sigma_{\mathrm{sc}}$ is field independent. ${ }^{20}$ We measured $\sigma_{\mathrm{sc}}$ as a function of the applied field at $1.7 \mathrm{~K}$ (see Fig. 3(b)). Each point was obtained by field cooling the sample from above $T_{\mathrm{c}}$ to $1.7 \mathrm{~K}$. First, $\sigma_{\mathrm{sc}}$ strongly increases with increasing magnetic field until reaching a maximum at $\mu_{0} H \simeq 0.03 \mathrm{~T}$ and then above $0.03 \mathrm{~T}$ stays nearly constant up to the highest field $(0.64 \mathrm{~T})$ investigated. Such a behavior is expected within the London model and is typical for polycrystalline high temperature superconductors (HTS's). ${ }^{21}$ The observed field dependence of $\sigma_{\mathrm{sc}}$ implies that for a reliable determination of the penetration depth the applied field must be larger than $\mu_{0} H=0.03 \mathrm{~T}$.

$\lambda(T)$ can be calculated within the local (London) approximation $(\lambda \gg \xi)$ by the following expression: ${ }^{19,22}$

$\frac{\lambda^{-2}\left(T, \Delta_{0, i}\right)}{\lambda^{-2}\left(0, \Delta_{0, i}\right)}=1+\frac{1}{\pi} \int_{0}^{2 \pi} \int_{\Delta(T, \varphi)}^{\infty}\left(\frac{\partial f}{\partial E}\right) \frac{E d E d \varphi}{\sqrt{E^{2}-\Delta_{i}(T, \varphi)^{2}}}$,

where $f=\left[1+\exp \left(E / k_{\mathrm{B}} T\right)\right]^{-1}$ is the Fermi function, $\varphi$ is the angle along the Fermi surface, and $\Delta_{i}(T, \varphi)=$ $\Delta_{0, i} \delta\left(T / T_{\mathrm{c}}\right) g(\varphi)\left(\Delta_{0, i}\right.$ is the maximum gap value at $\left.T=0\right)$. The temperature dependence of the gap is approximated by the expression $\delta\left(T / T_{\mathrm{c}}\right)=\tanh \left\{1.82\left[1.018\left(T_{\mathrm{c}} / T-1\right)\right]^{0.51}\right\}$ (Ref. 23), while $g(\varphi)$ describes the angular dependence of the gap and it is replaced by 1 for both an $s$-wave and an $s+$ $s$-wave gap, and $|\cos (2 \varphi)|$ for a $d$-wave gap. ${ }^{24}$

The temperature dependence of the penetration depth was analyzed using either a single gap or a two-gap model which is based on the so-called $\alpha$ model. This model was first discussed by Padamsee et $a .^{25}$ and later on was successfully used to analyze the magnetic penetration depth data in HTS's. ${ }^{23,26}$ According to the $\alpha$ model, the superfluid density is calculated 


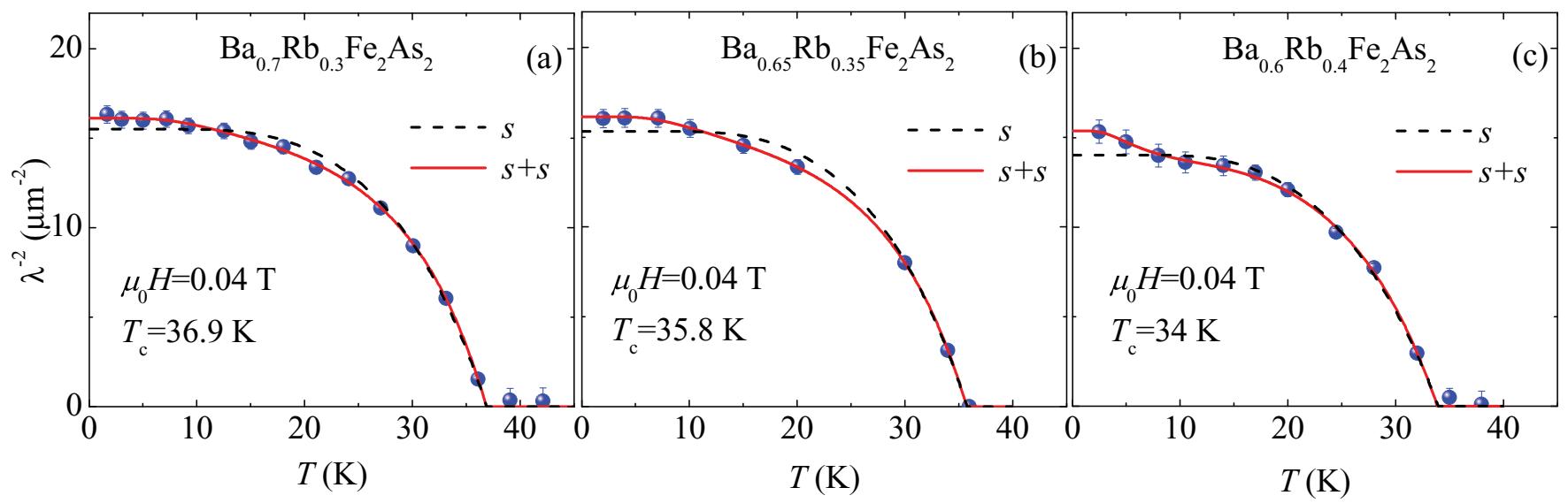

FIG. 4. (Color online) The temperature dependence of $\lambda^{-2}$ for $\mathrm{Ba}_{1-x} \mathrm{Rb}_{x} \mathrm{Fe}_{2} \mathrm{As}_{2}$, measured in an applied field of $\mu_{0} H=0.04 \mathrm{~T}$ : (a) $x=0.3$, (b) $x=0.35$, and (c) $x=0.4$. The dashed lines correspond to a single gap BCS $s$-wave model, whereas the solid ones represent a fit using a two-gap $(s+s)$-wave model.

for each component using Eq. (3) and then the contributions from the two components added together

$$
\frac{\lambda^{-2}(T)}{\lambda^{-2}(0)}=\omega_{1} \frac{\lambda^{-2}\left(T, \Delta_{0,1}\right)}{\lambda^{-2}\left(0, \Delta_{0,1}\right)}+\omega_{2} \frac{\lambda^{-2}\left(T, \Delta_{0,2}\right)}{\lambda^{-2}\left(0, \Delta_{0,2}\right)},
$$

where $\lambda^{-2}(0)$ is the penetration depth at zero temperature, $\Delta_{0, i}$ is the value of the $i$ th $(i=1,2)$ superconducting gap at $T=$ $0 \mathrm{~K}$, and $\omega_{i}$ is a weighting factor which measures their relative contributions to $\lambda^{-2}\left(\omega_{1}+\omega_{2}=1\right)$.

The results of the analysis for $\mathrm{Ba}_{1-x} \mathrm{Rb}_{x} \mathrm{Fe}_{2} \mathrm{As}_{2}(x=0.3$, $0.35,0.4)$ are presented in Fig. 4 . The dashed and the solid lines represent a fit to the data using an $s$-wave and a $s+s$-wave models, respectively. The analysis appears to rule out the simple $s$-wave model as an adequate description of $\lambda(T)$ for $\mathrm{Ba}_{1-x} \mathrm{Rb}_{x} \mathrm{Fe}_{2} \mathrm{As}_{2}(x=0.3,0.35,0.4)$. A $d$-wave gap symmetry was also tested, but was found to be inconsistent with the data. The two-gap $s+s$-wave scenario with a small gap $\Delta_{1}$ and a large gap $\Delta_{2}$, describes the experimental data remarkably well. The results of all samples extracted from the data analysis are summarized in Table I. A two-gap scenario is in line with the generally accepted view of multigap superconductivity in Fe-based HTS. ${ }^{5,6,8,27-29}$ The magnitudes of the large and the small gaps for $\mathrm{Ba}_{1-x} \mathrm{Rb}_{x} \mathrm{Fe}_{2} \mathrm{As}_{2}(x=0.3$, $0.35,0.4$ ) (see Table I) are in good agreement with the results of a previous report. ${ }^{5}$ There it was pointed out that most Fe-based HTS's exhibit two-gap superconducting behavior,

TABLE I. Summary of the parameters obtained for polycrystalline samples of $\mathrm{Ba}_{1-x} \mathrm{Rb}_{x} \mathrm{Fe}_{2} \mathrm{As}_{2}(x=0.3,0.35,0.4,1)$ by means of $\mu \mathrm{SR}$. The data for $x=1.0$ are taken from Ref. 10 .

\begin{tabular}{lcccl}
\hline \hline & $x=0.3$ & $x=0.35$ & $x=0.4$ & $x=1.0$ \\
\hline$T_{\mathrm{c}}(\mathrm{K})$ & 36.9 & 35.8 & 34 & 2.52 \\
$\Delta_{1}(\mathrm{meV})$ & $3.2(7)$ & $2.9(8)$ & $1.1(3)$ & $0.15(2)$ \\
$2 \Delta_{1} / k_{\mathrm{B}} T_{\mathrm{c}}$ & $2.0(5)$ & $1.9(4)$ & $0.8(6)$ & $1.4(2)$ \\
$\Delta_{2}(\mathrm{meV})$ & $9.2(3)$ & $8.8(3)$ & $7.5(2)$ & $0.49(4)$ \\
$2 \Delta_{2} / k_{\mathrm{B}} T_{\mathrm{c}}$ & $5.8(6)$ & $5.7(5)$ & $5.1(4)$ & $4.5(4)$ \\
$\omega_{1}$ & $0.19(5)$ & $0.21(4)$ & $0.15(3)$ & $0.36(3)$ \\
$\lambda(\mathrm{nm})$ & $249(15)$ & $250(17)$ & $255(9)$ & $267(5)$ \\
\hline \hline
\end{tabular}

characterized by a large gap with $2 \Delta / k_{\mathrm{B}} T_{\mathrm{c}}=7(2)$ and a small one with $2.5(1.5)$. To reach a more complete view of the superconducting properties of $\mathrm{Ba}_{1-x} \mathrm{Rb}_{x} \mathrm{Fe}_{2} \mathrm{As}_{2}$ as a function of the $\mathrm{Rb}$ composition (hole doping), we combined the present data with the previous $\mu \mathrm{SR}$ results on $\mathrm{RbFe}_{2} \mathrm{As}_{2}$ (Ref. 10) which presents the case of a naturally overdoped system. Figure 5 shows the small gap to $T_{c}$ ratio $2 \Delta_{1} / k_{\mathrm{B}} T_{\mathrm{c}}$, the large gap to $T_{c}$ ratio $2 \Delta_{2} / k_{\mathrm{B}} T_{\mathrm{c}}$, and the weight $\omega_{1}$ of the small gap to the superfluid density as a function of $\mathrm{Rb}$ concentration. The data for $\mathrm{RbFe}_{2} \mathrm{As}_{2}$ are taken from Ref. 10 . Interestingly, the ratio $2 \Delta_{2} / k_{\mathrm{B}} T_{\mathrm{c}}$ decreases with increasing $x$. On the other hand, the ratio $2 \Delta_{1} / k_{\mathrm{B}} T_{\mathrm{c}}$ for the small gap is essentially independent of $x$. In addition, the weighting factor $\omega_{1}$ is found to increase with increasing $x$. We note that in the optimally doped 122-system $\mathrm{Ba}_{1-x} \mathrm{~K}_{x} \mathrm{Fe}_{2} \mathrm{As}_{2}$ several bands cross the Fermi surface (FS) ${ }^{5-7}$ They consist of inner $(\alpha)$ and outer $(\beta)$ hole-like bands, both centered at the zone center $\Gamma$, and an electron-like band $(\gamma)$ centered at the $M$ point. The superconducting gap opened on the $\beta$ band was found to be smaller than those on the $\alpha$ and $\gamma$ bands. It was proposed that the enhanced interband scattering between the $\alpha$ and $\gamma$ bands might promote the kinetic process of pair scattering between these two FSs, leading to an increase of the pairing amplitude. ${ }^{30}$ Hole doping may cause a shift of the band bottom of the electron pockets above the Fermi level $E_{F}$. As a result, the interband scattering between $\alpha$ and $\gamma$ bands would diminish since the $\gamma$ band is in the unoccupied side and concomitantly the size of the $\alpha$ band is increased. According to ARPES results, a decrease of interband scattering will lead to a decrease of the pairing amplitude and the ratio $2 \Delta / k_{\mathrm{B}} T_{\mathrm{c}}$ in agreement with the results presented in Fig. 5(a). These results suggest the possible role of interband processes in optimally hole-doped iron-based 122 superconductors. ${ }^{6,30}$

One of the most interesting results of $\mu \mathrm{SR}$ investigations in HTS's is the observation of a remarkable proportionality between $T_{\mathrm{c}}$ and the zero-temperature relaxation rate $\sigma(0) \propto 1 / \lambda^{2}(0)$ (Uemura relation). ${ }^{31}$ This relation $T_{\mathrm{c}}(\sigma)$, which seems to be generic for various families of cuprate HTS's, has the features that upon increasing the charge carrier doping $T_{\mathrm{c}}$ first increases linearly in the underdoped region 


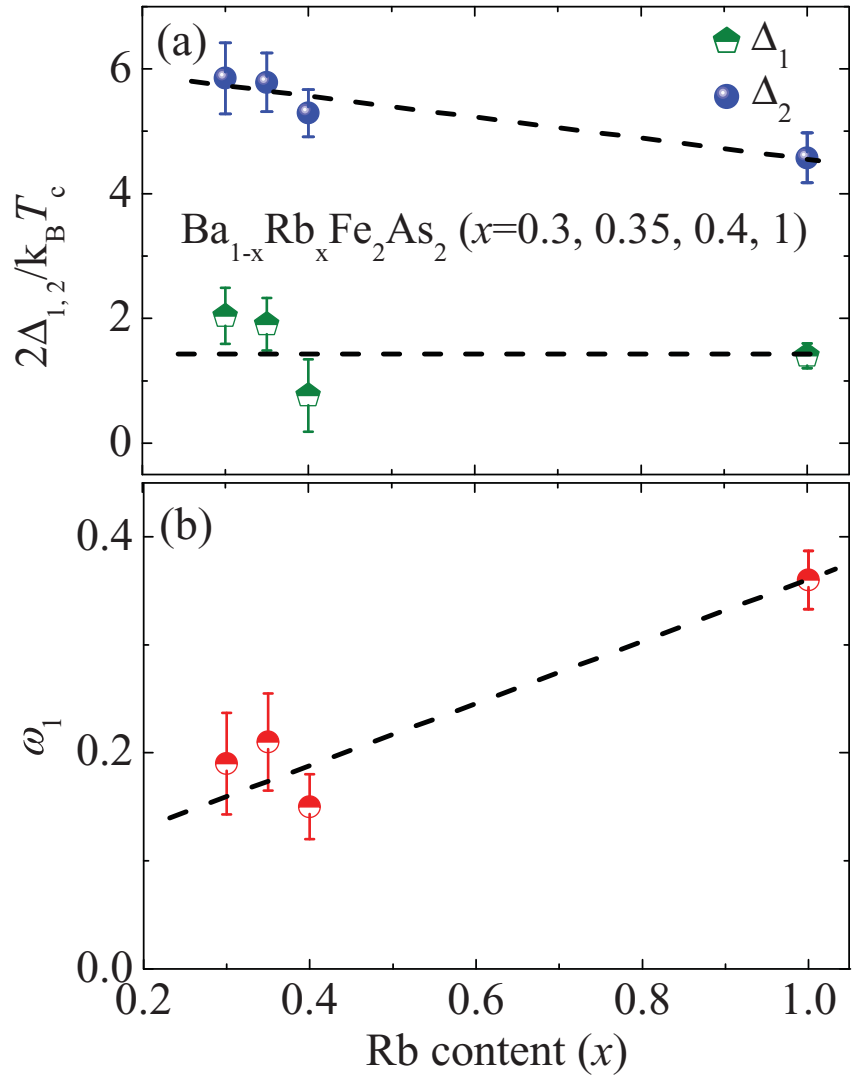

FIG. 5. (Color online) (a) Superconducting gap to $T_{c}$ ratios $2 \Delta_{1,2} / k_{\mathrm{B}} T_{\mathrm{c}}$ and (b) the contribution $\omega_{1}$ of the small gap to the superfluid density as a function of the $\mathrm{Rb}$ composition for $\mathrm{Ba}_{1-x} \mathrm{Rb}_{x} \mathrm{Fe}_{2} \mathrm{As}_{2}$ $(x=0.3,0.35,0.4,1.0)$. The measurements were performed in an applied magnetic field of $\mu_{0} H=0.04 \mathrm{~T}$. The data for $\mathrm{RbFe}_{2} \mathrm{As}_{2}$ are taken from Ref. 10. The dashed lines represent guides to the eyes.

(Uemura line), then saturates, and finally is suppressed for high carrier doping. The initial linear trend of the Uemura relation indicates that for these unconventional HTS's the ratio $T_{c} / E_{F}$ ( $E_{\mathrm{F}}$ is the Fermi energy) is up to two orders of magnitude larger than for conventional BCS superconductors. Figure 6 shows $T_{\mathrm{c}}$ vs $\sigma(0)$ plot for various hole- and electrondoped high $T_{\mathrm{c}}$ Fe-based superconductors (after Ref. 28), including the present results. The solid line shows the Uemura relation in hole-doped cuprates ${ }^{31}$ and the dashed line corresponds to electron-doped cuprates as observed by Shengelaya et $a l{ }^{32}$ The Uemura relation for Fe-based superconductors was already discussed in Ref. 28. Here, we demonstrate that the data points for $\mathrm{Ba}_{1-x} \mathrm{Rb}_{x} \mathrm{Fe}_{2} \mathrm{As}_{2}(x=0.3,0.35,0.4)$ are located in the Uemura plot close to those of the other Fe-based superconductors. On the other hand, for naturally fully overdoped $\mathrm{RbFe}_{2} \mathrm{As}_{2}$, the ratio $T_{c} / \sigma(0)$ is strongly reduced. The small value of ratio $T_{c} / \sigma(0)$ is characteristic for conventional superconductors. For comparison the point for conventional BCS superconductor $\mathrm{Nb}$ is also shown on the Uemura plot. This suggests that superconductivity in the compound $\mathrm{RbFe}_{2} \mathrm{As}_{2}$ has a more conventional character. Additional experiments are in progress to clarify this point.

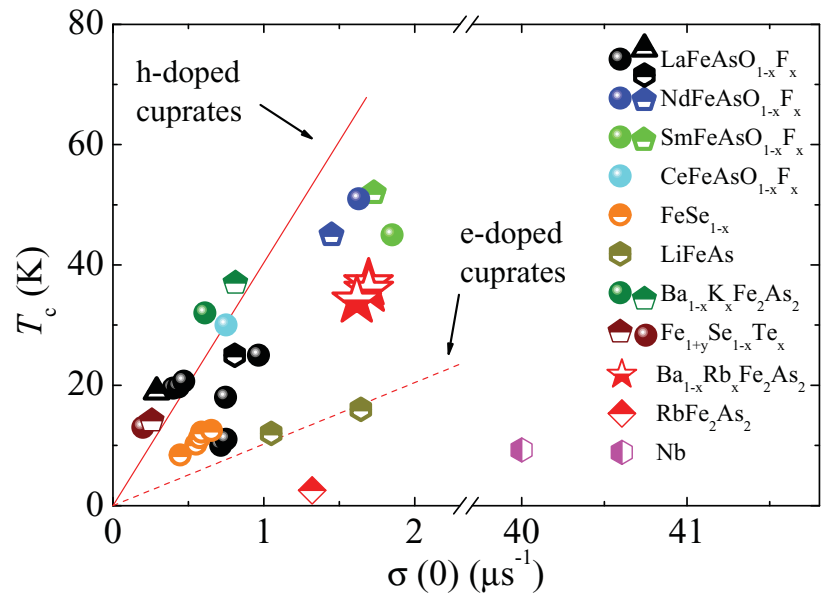

FIG. 6. (Color online) Uemura plot for hole- and electron-doped high $T_{\mathrm{c}}$ Fe-based superconductors (after Ref. 28). The Uemura relation observed for underdoped cuprates is also shown (solid line for hole doping and dashed line for electron doping) (after Ref. 32). The point for conventional BCS superconductor $\mathrm{Nb}$ is also shown. Data points for the pnictides are taken from Refs. 27-29, 33-39. The stars show the data for $\mathrm{Ba}_{1-x} \mathrm{Rb}_{x} \mathrm{Fe}_{2} \mathrm{As}_{2}(x=0.3,0.35,0.4)$ obtained in this work. The point for $\mathrm{RbFe}_{2} \mathrm{As}_{2}$ is taken from Ref. 10 .

\section{SUMMARY AND CONCLUSION}

In summary, we performed transverse-field $\mu$ SR measurements of the magnetic penetration depth $\lambda$ on polycrystalline samples of the iron-based HTS's $\mathrm{Ba}_{1-x} \mathrm{Rb}_{x} \mathrm{Fe}_{2} \mathrm{As}_{2}(x=0.3$, $0.35,0.4)$. The values of the superconducting transition temperature $T_{\mathrm{c}}$ and the zero temperature values of $\lambda$ were estimated to be $T_{\mathrm{c}}=36.9,35.8,34 \mathrm{~K}$ and $\lambda(0)=249(15)$, 250(17), 255(9) $\mathrm{nm}$ for $x=0.3,0.35$, and 0.4 , respectively. The temperature dependence of $\lambda$ is well described by a two-gap $s+s$-wave scenario with gap values similar to $\mathrm{Ba}_{1-x} \mathrm{~K}_{x} \mathrm{Fe}_{2} \mathrm{As}_{2}$ (Refs. 5 and 8). ARPES investigations of $\mathrm{Ba}_{1-x} \mathrm{~K}_{x} \mathrm{Fe}_{2} \mathrm{As}_{2}$ revealed that the large gap opens on the inner hole-like Fermi surface ( $\alpha$ band) centered at the $\Gamma$ point and on the electron-like FS ( $\gamma$ band) centered at the $M$ point (tetragonal structure notations), while the small gap opens on the outer hole-like band $(\beta)$ of the $\Gamma$ point. ${ }^{30} \mathrm{We}$ found that the large gap to $T_{\mathrm{c}}$ ratio $2 \Delta_{2} / k_{\mathrm{B}} T_{\mathrm{c}}$ decreases with increasing $\mathrm{Rb}$ content $x$. On the other hand, for the small gap opening on the $\alpha$ and $\gamma$ bands, the ratio $2 \Delta_{1} / k_{\mathrm{B}} T_{\mathrm{c}}$ is practically independent of $x$. In addition, the contribution of the small gap $\omega_{1}$ to the total superfluid density increases with increasing $x$. These results may be interpreted by assuming a disappearance of the electron pocket from the Fermi surface upon the high hole doping, resulting in a suppression of the scattering processes between the $\alpha$ and $\gamma$ bands. This might cause the reduction of $T_{\mathrm{c}}$ for the overdoped $\mathrm{RbFe}_{2} \mathrm{As}_{2}$. We also performed zero-field $\mu \mathrm{SR}$ experiments and found no evidence of either static or fluctuating magnetism, implying that the spin-density wave ordering of the Fe moments is completely suppressed upon $\mathrm{Rb}$ doping. The absence of field induced magnetism in the investigated compounds is also demonstrated. Finally, the correlation between $T_{\mathrm{c}}$ and the zero-temperature relaxation rate $\sigma(0) \propto 1 / \lambda^{2}(0)$ is discussed for the samples $\mathrm{Ba}_{1-x} \mathrm{Rb}_{x} \mathrm{Fe}_{2} \mathrm{As}_{2}(x=0.3,0.35,0.4,1)$ using the Uemura classification scheme. 


\section{ACKNOWLEDGMENTS}

Part of this work was performed at the Swiss Muon Source, Paul Scherrer Institut, Villigen, Switzerland. This work was supported by the Swiss National Science Foundation, the
SCOPES Grant No. IZ73Z0_128242, the NCCR Project MaNEP, the EU Project CoMePhS, and the Georgian National Science Foundation Grant No. GNSF/ST08/4-416. *zurabgug@physik.uzh.ch

${ }^{1}$ Y. Kamihara, T. Watanabe, M. Hirano, and H. Hosono, J. Am. Chem. Soc. 130, 3296 (2008).

${ }^{2}$ Z. A. Ren, W. Lu, J. Yang, W. Yi, X. L. Shen, Z. C. Li, G. C. Che, X. L. Dong, L. L. Sun, F. Zhou, and Z. X. Zhao, Chin. Phys. Lett. 25, 2215 (2008).

${ }^{3}$ M. Rotter, M. Tegel, and D. Johrendt, Phys. Rev. Lett. 101, 107006 (2008).

${ }^{4}$ Z. Bukowski, S. Weyeneth, R. Puzniak, J. Karpinski, and B. Batlogg, Physica C 470, S328 (2010).

${ }^{5}$ D. V. Evtushinsky, D. S. Inosov, V. B. Zabolotnyy, M. S. Viazovska, R. Khasanov, A. Amato, H.-H. Klauss, H. Luetkens, Ch. Niedermayer, G. L. Sun, V. Hinkov, C. T. Lin, A. Varykhalov, A. Koitzsch, M. Knupfer, B. Büchner, A. A. Kordyuk, and S. V. Borisenko, New J. Phys. 11, 055069 (2009).

${ }^{6}$ H. Ding, P. Richard, K. Nakayama, K. Sugawara, T. Arakane, Y. Sekiba, A. Takayama, S. Souma, T. Sato, T. Takahashi, Z. Wang, X. Dai, Z. Fang, G. F. Chen, J. L. Luo, and N. L. Wang, Europhys. Lett. 83, 47001 (2008).

${ }^{7}$ V. B. Zabolotnyy, D. V. Evtushinsky, A. A. Kordyuk, D. S. Inosov, A. Koitzsch, A. V. Boris, G. L. Sun, C. T. Lin, M. Knupfer, B. Büchner, A. Varykhalov, R. Follath, and S. V. Borisenko, Nature (London) 457, 569 (2009).

${ }^{8}$ R. Khasanov, D. V. Evtushinsky, A. Amato, H.-H. Klauss, H. Luetkens, Ch. Niedermayer, B. Büchner, G. L. Sun, C. T. Lin, J. T. Park, D. S. Inosov, and V. Hinkov, Phys. Rev. Lett. 102, 187005 (2009).

${ }^{9}$ J. E. Sonier, J. H. Brewer, and R. F. Kiefl, Rev. Mod. Phys. 72, 769 (2000).

${ }^{10}$ Z. Shermadini, J. Kanter, C. Baines, M. Bendele, Z. Bukowski, R. Khasanov, H.-H. Klauss, H. Luetkens, H. Maeter, G. Pascua, B. Batlogg, and A. Amato, Phys. Rev. B 82, 144527 (2010).

${ }^{11}$ Q. Huang, Y. Qiu, W. Bao, M. A. Green, J. W. Lynn, Y. C. Gasparovic, T. Wu, G. Wu, and X. H. Chen, Phys. Rev. Lett. 101, 257003 (2008).

${ }^{12}$ M. S. Torikachvili, S. L. Bud'ko, N. Ni, and P. C. Canfield, Phys. Rev. Lett. 101, 057006 (2008).

${ }^{13}$ C. F. Miclea, M. Nicklas, H. S. Jeevan, D. Kasinathan, Z. Hossain, H. Rosner, P. Gegenwart, C. Geibel, and F. Steglich, Phys. Rev. B 79, 212509 (2009).

${ }^{14}$ J. Zhao, Q. Huang, C. de la Cruz, S. Li, J. W. Lynn, Y. Chen, M. A. Green, G. F. Chen, G. Li, Z. Li, J. L. Luo, N. L. Wang, and P. Dai, Nat. Mater. 7, 953 (2008).

${ }^{15} \mathrm{R}$. Kubo and T. Toyabe, Magnetic Resonance and Relaxation (North Holland, Amsterdam, 1967).

${ }^{16}$ R. Khasanov, A. Maisuradze, H. Maeter, A. Kwadrin, H. Luetkens, A. Amato, W. Schnelle, H. Rosner, A. Leithe-Jasper, and H.-H. Klauss, Phys. Rev. Lett. 103, 067010 (2009).

${ }^{17}$ T. J. Williams, A. A. Aczel, E. Baggio-Saitovitch, S. L. Bud'ko, P. C. Canfield, J. P. Carlo, T. Goko, H. Kageyama, A. Kitada, J. Munevar, N. Ni, S. R. Saha, K. Kirschenbaum, J. Paglione, D. R.
Sanchez-Candela, Y. J. Uemura, and G. M. Luke, Phys. Rev. B 82 , 094512 (2010).

${ }^{18}$ J. E. Sonier, W. Huang, C. V. Kaiser, C. Cochrane, V. Pacradouni, S. A. Sabok-Sayr, M. D. Lumsden, B. C. Sales, M. A. McGuire, A. S. Sefat, and D. Mandrus, Phys. Rev. Lett. 106, 127002 (2011).

${ }^{19}$ The data were analyzed with the fitting package MUSRFIT developed by A. Suter and B. Wojek (see http://lmu.web.psi.ch/facilities/ software/musrfit/user/MUSR/WebHome.html). The fitting of Eq. (3) was performed using the additional library BMW developped by B. M. Wojek.

${ }^{20}$ E. H. Brandt, Phys. Rev. B 37, 2349 (1988).

${ }^{21}$ B. Pümpin, H. Keller, W. Kündig, W. Odermatt, I. M. Savić, J. W. Schneider, H. Simmler, P. Zimmermann, E. Kaldis, S. Rusiecki, Y. Maeno, and C. Rossel, Phys. Rev. B 42, 8019 (1990).

${ }^{22} \mathrm{M}$. Tinkham, Introduction to Superconductivity (Krieger, Malabar, FL, 1975).

${ }^{23}$ A. Carrington and F. Manzano, Physica C 385, 205 (2003).

${ }^{24}$ M. H. Fang, H. M. Pham, B. Qian, T. J. Liu, E. K. Vehstedt, Y. Liu, L. Spinu, and Z. Q. Mao, Phys. Rev. B 78, 224503 (2008).

${ }^{25}$ H. Padamsee, J. E. Neighbor, and C. A. Shiffman, J. Low Temp. Phys. 12, 387 (1973).

${ }^{26}$ R. Khasanov, A. Shengelaya, A. Maisuradze, F. La Mattina, A. Bussmann-Holder, H. Keller, and K. A. Müller, Phys. Rev. Lett. 98, 057007 (2007)

${ }^{27}$ R. Khasanov, K. Conder, E. Pomjakushina, A. Amato, C. Baines, Z. Bukowski, J. Karpinski, S. Katrych, H.-H. Klauss, H. Luetkens, A. Shengelaya, and N. D. Zhigadlo, Phys. Rev. B 78, 220510 (2008).

${ }^{28}$ M. Bendele, S. Weyeneth, R. Puzniak, A. Maisuradze, E. Pomjakushina, K. Conder, V. Pomjakushin, H. Luetkens, S. Katrych, A. Wisniewski, R. Khasanov, and H. Keller, Phys. Rev. B 81, 224520 (2010).

${ }^{29}$ H. Luetkens, H.-H. Klauss, R. Khasanov, A. Amato, R. Klingeler, I. Hellmann, N. Leps, A. Kondrat, C. Hess, A. Köhler, G. Behr, J. Werner, and B. Büchner, Phys. Rev. Lett. 101, 097009 (2008).

${ }^{30}$ T. Sato, K. Nakayama, Y. Sekiba, P. Richard, Y.-M. Xu, S. Souma, T. Takahashi, G. F. Chen, J. L. Luo, N. L. Wang, and H. Ding, Phys. Rev. Lett. 103, 047002 (2009).

${ }^{31}$ Y. J. Uemura, G. M. Luke, B. J. Sternlieb, J. H. Brewer, J. F. Carolan, W. N. Hardy, R. Kadono, J. R. Kempton, R. F. Kiefl, S. R. Kreitzman, P. Mulhern, T. M. Riseman, D. Ll. Williams, B. X. Yang, S. Uchida, H. Takagi, J. Gopalakrishnan, A. W. Sleight, M. A. Subramanian, C. L. Chien, M. Z. Cieplak, G. Xiao, V. Y. Lee, B. W. Statt, C. E. Stronach, W. J. Kossler, and X. H. Yu, Phys. Rev. Lett. 62, 2317 (1989).

${ }^{32}$ A. Shengelaya, R. Khasanov, D. G. Eshchenko, D. Di Castro, I. M. Savic, M. S. Park, K. H. Kim, S. I. Lee, K. A. Müller, and H. Keller, Phys. Rev. Lett. 94, 127001 (2005).

${ }^{33}$ H. Luetkens, H.-H. Klauss, M. Kraken, F. J. Litterst, T. Dellmann, R. Klingeler, C. Hess, R. Khasanov, A. Amato, C. Baines, 
M. Kosmala, O. J. Schumann, M. Braden, J. Hamann-Borrero, N. Leps, A. Kondrat, G. Behr, J. Werner, and B. Büchner, Nat. Mater. 8, 305 (2009).

${ }^{34}$ H. Kim, C. Martin, R. T. Gordon, M. A. Tanatar, J. Hu, B. Qian, Z. Q. Mao, Rongwei Hu, C. Petrovic, N. Salovich, R. Giannetta, and R. Prozorov, Phys. Rev. B 81, 180503(R) (2010).

${ }^{35}$ S. Takeshita and R. Kadono, New J. Phys. 11, 035006 (2009).

${ }^{36}$ J. P. Carlo, Y. J. Uemura, T. Goko, G. J. MacDougall, J. A. Rodriguez, W. Yu, G. M. Luke, P. Dai, N. Shannon, S. Miyasaka,
S. Suzuki, S. Tajima, G. F. Chen, W. Z. Hu, J. L. Luo, and N. L. Wang, Phys. Rev. Lett. 102, 087001 (2009).

${ }^{37}$ R. Khasanov, H. Luetkens, A. Amato, H.-H. Klauss, Z.-A. Ren, J. Yang, W. Lu, and Z.-X. Zhao, Phys. Rev. B 78, 092506 (2008).

${ }^{38}$ R. Khasanov, M. Bendele, A. Amato, K. Conder, H. Keller, H.-H. Klauss, H. Luetkens, and E. Pomjakushina, Phys. Rev. Lett. 104, 087004 (2010).

${ }^{39}$ F. L. Pratt, P. J. Baker, S. J. Blundell, T. Lancaster, H. J. Lewtas, P. Adamson, M. J. Pitcher, D. R. Parker, and S. J. Clarke, Phys. Rev. B 79, 052508 (2009). 